\title{
Fractional Fourier domain hopped communication method based on chirp modulation for underwater acoustic channels
}

\author{
Xingbin $\mathrm{Tu}^{1,2}$, Xiaomei $\mathrm{Xu}^{1,2, *}$, Zheguang Zou ${ }^{1,2}$, Liangliang Yang ${ }^{1,2}$, and Jianming $\mathrm{Wu}^{1,2}$ \\ 1. College of Ocean and Earth Science, Xiamen University, Xiamen 361102, China; \\ 2. Key Laboratory of Underwater Acoustic Communication and Marine Information Technology, \\ Ministry of Education, Xiamen 361005, China
}

\begin{abstract}
To improve the data rate of underwater acoustic frequency-hopped communications, frequency hopping is applied to different orders of fractional Fourier domain (FrFD), to enable non-intrusive, bandwidth-limited acoustic communications. An FrFD frequency-hopped communication method based on chirp modulation, namely multiple chirp shift keying-FrFD hopping (MCSK-FrFDH), is proposed for underwater acoustic channels. Validated by both simulations and experimental results, this method can reach a bandwidth efficiency twice more than conventional frequency-hopped methods with the same data rate and anti-multipath capability, suggesting that the proposed method achieves a better performance than the traditional frequencyhopped communication in underwater acoustic communication channels. Results also show that in practical scenarios, the MCSKFrFDH system with longer symbol length performs better at the low signal-to-noise ratio (SNR), while the system with larger frequency sweeping range performs better at a high SNR.
\end{abstract}

Keywords: underwater acoustic communication, fractional Fourier domain (FrFD), frequency hopping, chirp signal.

DOI: $10.21629 /$ JSEE.2017.03.05

\section{Introduction}

Frequency hopping is one of the most robust techniques to overcome severe multipath effect for underwater acoustic communications, particularly in shallow waters, since it works with multiple frequency shift keying (MFSK), which is based on energy detection, for modulation $[1,2]$. Though the frequency-hopped MFSK has a relatively lower bandwidth efficiency and data rates comparing to phase shift keying (PSK) based coherent communication schemes, it still plays a crucial role in reliable acoustic communications in shallow-water environments [3]. Thereby, improving bandwidth efficiency is a significant

\footnotetext{
Manuscript received December 10, 2015.

*Corresponding author.

This work was supported by the National Natural Science Foundation of China (41376040; 41676024).
}

task for designing reliable high-speed frequency-hopped acoustic communication systems.

A common approach to increase the bandwidth efficiency for frequency-hopped systems is to combine frequency hopping with other techniques such as multi-carrier modulation or frequency division multiplexing (FDM). The idea is to convert the transmitted data stream from serial to parallel, i.e. transforming from one carrier band to multiple sub-carrier bands. For example, Beaujean [4] reported a frequency-hopped frequency division multiplexed (FH-FDM) system working within a waterdepth of $3 \mathrm{~m}$ and a range of $120 \mathrm{~m}$, taking into account of $9.31 \mathrm{~ms}$ reverberation and $1.5 \mathrm{~Hz}$ Doppler spreading. It achieved a data rate of 5272 bps and a bit error rate of less than $0.14 \%$, but the required bandwidth was up to $27 \mathrm{kHz}$.

Another approach to increase bandwidth efficiency for frequency-hopped systems is to apply independent multisource parallel transmission. It sends data with more than one transmitter and requires spatial diversity for receivers, as the received signal is the superposition of signals from all sources. For example, Hou [5] designed a frequency-hopped code division multiple accessed (FHCDMA) system, where each sender was assigned one frequency-hopped pattern chosen from the mutually orthogonal frequency-hopped sequence family, enabling it to independently access shared underwater acoustic channel with zero access delay. Besides, it was insensitive to nearfar effect and no power control was needed.

Since these methods have requirements for either transmitters or receivers, which are not widely-applicable, underwater acoustic communications are calling for new methods with no restriction from hardware. In recent years, with the development of fractional Fourier transform $(\mathrm{FrFT})$ and its fast discrete algorithm [6,7], FrFT has exhibited some advantages in both wireless and underwater acoustic communications. On one hand, according to 
the property of chirp basis decomposition, the energy of a chirp signal can be concentrated at one point in fractional Fourier domain (FrFD) with FrFT. On the other hand, as a broadband signal, a chirp signal may cope with frequency selective channels effectively. Meanwhile, it has a large tolerance of Doppler shift when combining with scaling characteristics, so no Doppler compensation algorithms are required [8]. Therefore, applying chirp signals to underwater acoustic communication together with FrFT for demodulation can be a solution to improving frequencyhopped communication. For instance, Sha et al. $[9,10]$ proposed a terrestrial wireless communication scheme using different chirp signals for modulation and associated FrFDs for demodulation. This method is reliable when the FrFD spectra at non-optimal orders do not affect those at the optimal order, but it requires significant differences between any two normalized frequency modulation rates, which may be difficult to realize in bandwidth-limited underwater acoustic channels.

This paper proposes a scheme for underwater acoustic communications based on multiple chirp shift keyingFrFD hopping (MCSK-FrFDH) within two FrFDs. Chirp pairs with opposite frequency modulation rates are used for modulation to maximize the difference of frequency modulation rate. At the transmitter side, each chirp signal hops in different FrFDs with a preset pattern; while at the receiver side, signals are demodulated in the two associated FrFDs using FrFT. With this design, bandwidth utilization and the data rate are boosted, and the ability of anti-multipath from the traditional frequency-hopped method is still retained.

\section{FrFT and FrFD}

FrFT is an extension of traditional Fourier transform, whose earliest research could be traced back to 1929 . Since FrFT is a linear transformation, the cross terms can be removed in time-frequency distribution [11]. With FrFT, time-domain and frequency-domain signal characteristics show up in FrFD simultaneously. Denote FrFD as $u$ domain, and the $p$-order FrFT of signal $s(t)$ can be expressed as

$$
\left(\mathcal{F}^{p} s\right)(u)=\int_{-\infty}^{+\infty} K_{p}(t, u) s(t) \mathrm{d} t
$$

where

$$
\begin{gathered}
K_{p}(t, u)= \\
\left\{\begin{array}{l}
\sqrt{\frac{1-\mathrm{j} \cot \alpha}{2 \pi}} \operatorname{expj}\left(\frac{t^{2}+u^{2}}{2} \cot \alpha-t u \csc \alpha\right), \\
\alpha \neq n \pi \\
\delta(t-u), \quad \alpha=2 n \pi \\
\delta(t+u), \quad \alpha=(2 n \pm 1) \pi
\end{array}\right.
\end{gathered}
$$

is the FrFT's transform kernel, and $\alpha=p \pi / 2$ is the rotation angle. Specially, when $p=1$, the term $K_{p}(t, u)$ simplifies to $(1 / 2 \pi)^{1 / 2} \exp (-\mathrm{j} u t)$, turning $u$ domain to frequency domain and FrFT to the traditional Fourier transform.

One of the most important characteristics of FrFT is that the energy of a chirp signal may focus on one point with FrFT due to the property of chirp basis decomposition (see Fig. 1).

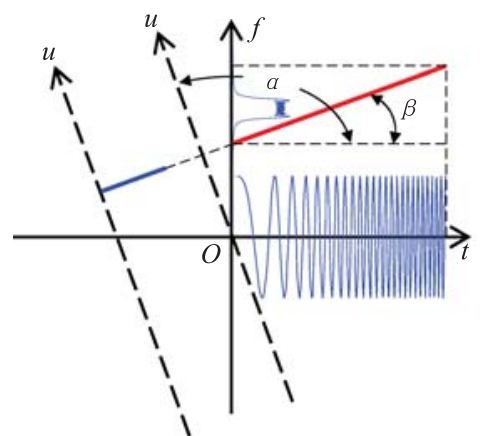

Fig. 1 Time-frequency graph of a chirp signal with its projections in different domains

The red line denotes the time-frequency curve of a chirp signal with normalized center frequency $f_{c}^{\prime}$ and normalized frequency modulation rate $\mu^{\prime}$ (for simplification, the variables with superscript ' denote the normalized variables). Its projection on $t$-axis $(p=0)$ is the time-domain signal and its projection on $f$-axis $(p=1)$ is the frequencydomain signal. When the rotation angle $\alpha$ is orthogonal to $\beta$ (the angle between the time-frequency curve and $t$-axis), i.e. $\alpha=\beta+\pi / 2$, the projection on $u$-axis shrinks to one point. Then, the optimal order $p_{0}$ satisfies that

$$
\mu^{\prime}=\tan \beta=\tan (\alpha-\pi / 2)=-\cot \left(p_{0} \pi / 2\right) .
$$

Correspondingly, the coordinate on $u$-axis is

$$
u_{0}=f_{c}^{\prime} \sin \left(p_{0} \pi / 2\right) .
$$

\section{Theory of MCSK-FrFDH}

There are some advantages for underwater acoustic frequency-hopped communication, including antimultipath, anti-interference and concealment. However, some disadvantages, such as low data rate and poor performance at the low signal-to-noise ratio (SNR), cannot be neglected. Since a chirp signal has the capacity for large Doppler tolerance and processing gain, chirp based frequency hopping is widely used in underwater acoustic communications [12]. Combining with FrFT of different orders, chirp based frequency hopping can be realized in different FrFDs. If we make the energy of modulated signals located in the same frequency-domain range 
as the traditional frequency hopping when extending it to FrFD hopping, the bandwidth utilization and data rate can be improved without losing its advantages mentioned above. Here, an important work is to establish the theory of MCSK-FrFDH by analogy.

In MFSK-FH modulation, the frequencies of frequencyhopped carrier hop in different sub-bands. Meanwhile the carrier is modulated according to MFSK in each sub-band. The modulation of MCSK-FrFDH system is similar to that. The FrFD frequencies hop in different FrFD sub-bands while the carrier is modulated according to MCSK in each FrFD sub-band. According to (3), once the frequency modulation rate of any chirp signal is confirmed, the optimal order is confirmed and the energy will not be concentrated on one point at other orders. For this reason, chirp signals with different frequency modulation rates can be used for FrFD hopping and do not interact with each other.

Note that the energy of a chirp signal does not concentrate in non-optimal order FrFDs. Instead, it expands to a certain bandwidth according to Parseval's theorem [13]. Consider two chirp signals with frequency modulation rates $\mu_{1}^{\prime}, \mu_{2}^{\prime}$ and optimal orders $p_{1}, p_{2}$. When their time-frequency curves are mutually orthogonal, the energy of chirp signal with $\mu_{1}^{\prime}\left(\mu_{2}^{\prime}\right)$ is expanded maximally and its amplitude is declined to the minimum in $p_{2}$-order $\left(p_{1}\right.$ order) FrFD. Accordingly, its FrFD spectrum has no influence on that of the chirp signal with $\mu_{2}^{\prime}\left(\mu_{1}^{\prime}\right)$. Actually, it is difficult to generate two mutually orthogonal chirp signals in underwater acoustic channels due to the restriction of transmitters and receivers. The best choice is to use chirp signals with opposite frequency modulation rates, to maximize the difference between them and reduce the interference, which is the main reason of choosing only two FrFDs for FrFD hopping. Moreover, the severe multipath delay and Doppler shift of underwater acoustic channels may shift FrFD spectrum and decrease the demodulation performance. Therefore, delay and Doppler shift estimation techniques should be adopted to eliminate their influence [14].

\subsection{MCSK-FrFDH system}

Fig. 2 shows the flowchart of MCSK-FrFDH system proposed in this paper. As seen in Fig. 2, the MCSK-FrFDH system is similar to the traditional MFSK-FH system, which means no auxiliary device is needed. The key differences between these two systems are modulator and demodulator, both of which are based on chirp signals in the MCSK-FrFDH system. Besides, the concept of frequency hopping is replaced with FrFD hopping, and the traditional frequency-domain filter is replaced with the FrFD filter.

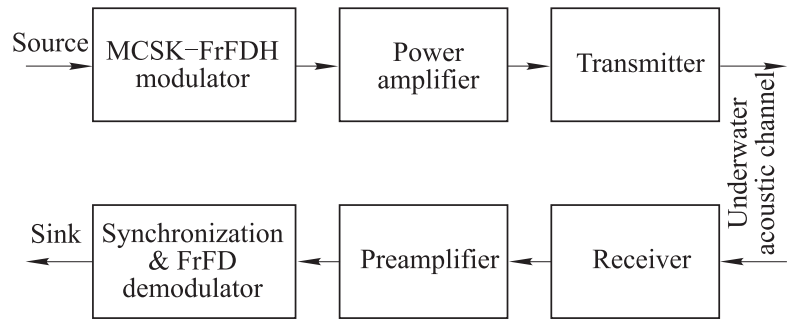

Fig. 2 Structure of an MCSK-FrFDH system

\subsection{Technical parameters of MCSK-FrFDH}

\subsubsection{Dimensional normalization}

The discrete data should be dimensional normalized before FrFT. Reference [15] presented two methods for dimensional normalization, namely discrete scaling transform method and data zero-padding/interception method. In this paper, we choose the former for normalization processing. Let $T_{s}$ designate the observation time of signal and $f_{s}$ the sample rate, then the scale factor $S$ and the normalized initial frequency interval are expressed as

$$
\begin{aligned}
& S=\sqrt{T_{s} / f_{s}} \\
& \Delta f^{\prime}=\Delta f S .
\end{aligned}
$$

\subsubsection{FrFD hopped pattern}

The FrFD hopped pattern represents the variation of carrier FrFD frequencies (see Fig. 3). In the time domain, the MCSK signal is composed of time-domain-FrFD arrays. The duration for each FrFD frequency is $T_{s}$ and each FrFD frequency lies in a distinguishable FrFD sub-band within different symbols. In the frequency domain, the frequencies in different symbols may even overlap. Let $f_{i}(t)$ designate the frequency of FrFD hopped sequences at moment $t, \Delta f$ the initial frequency interval of chirp signals, $\varphi_{k}$ the initial phase, $M$ the size of FrFD frequencies in each FrFD sub-band (usually a power of 2) and $m(t)$ the transmitted data, then the transmitted signal of MCSK-FrFDH system is

$$
s(t)=m(t) \cos \left\{2 \pi\left[\left(f_{i}(t)+n \Delta f\right) t+\frac{1}{2} \mu t^{2}\right]+\varphi_{k}\right\}
$$

where $n=0,1, \ldots, M-1$.

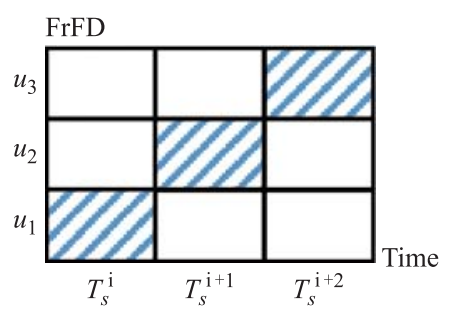

(a) FrFD frequency-hopped pattern of chirp signals with positive frequency modulation rate 


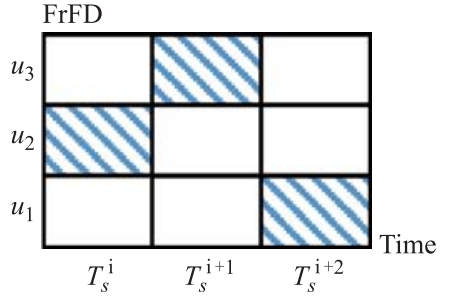

(b) FrFD frequency-hopped pattern of chirp signals with negative frequency modulation rate

Fig. 3 FrFD frequency-hopped patterns

\subsubsection{Minimum FrFD hopped interval and minimum} initial frequency interval

For $p_{0}$-order FrFD hopping, the minimum FrFD hopped interval $\Delta u$ should be no less than FrFD resolution $u_{d}$, and the FrFD spectral nulls should fall on the FrFD spectral peaks of adjacent bands, as Fig. 4 shows.

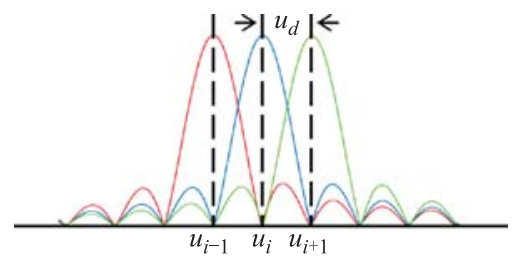

Fig. 4 Selection of minimum FrFD stepped-frequency interval

To ensure the orthogonality of FrFD frequencies, $\Delta u$ and $u_{d}$ should satisfy

$$
\Delta u=k u_{d}, \quad k=1,2, \ldots
$$

Similar to (4), $\Delta u$ can be expressed as

$$
\Delta u=\Delta f^{\prime} \sin \left(p_{0} \pi / 2\right) .
$$

By substituting (6) and (9) to (8), $\Delta u$ can be transformed into the minimum initial frequency interval $\Delta f$, i.e.

$$
\Delta f=\frac{k u_{d}}{S \sin \left(p_{0} \pi / 2\right)}, \quad k=1,2, \ldots
$$

\subsubsection{FrFD sub-band}

Similar to the relationship between frequency and FrFD frequency in (4), FrFD frequency band of MCSK-FrFDH can also be converted from frequency band correspondingly. Denote the frequency range from $f_{L}$ to $f_{H}$, hop count $H$, hopping sequence $h s$ and chirp bandwidth $B_{c}$, then we get

$$
f_{H}-f_{L}=H M \Delta f+B_{c} .
$$

For the $h$ th $(h=0,1, \ldots, H-1)$ hopping sequence, its initial frequency sub-band is from $\left[f_{L}+h s(h) M \Delta f\right]$ to $\left[f_{L}+h s(h) M \Delta f+M \Delta f\right]$.
Accordingly, the lower bound $u_{L}$ and upper bound $u_{H}$ of FrFD frequency band meet that

$$
u_{H}-u_{L}=H M \Delta u+B_{c} S \sin \left(p_{0} \pi / 2\right) .
$$

For the $h$ th FrFD hopping sequence, its FrFD frequency sub-band is from $\left[f_{L}+h s(h) M \Delta f\right] S \sin \left(p_{0} \pi / 2\right)$ to $\left[f_{L}+\right.$ $h s(h) M \Delta f+M \Delta f] S \sin \left(p_{0} \pi / 2\right)$.

According to these equations and requirements for antimultipath capability and data rate, we can calculate the most appropriate system parameters such as hopped count $H$, radix of MCSK $M$, initial frequency interval $\Delta f$ and frequency modulation rate $B_{c} / T_{s}$.

\section{Performance simulation}

\subsection{Modeling of underwater acoustic channel}

Underwater acoustic channel models can reflect the features of sound waves propagating in the ocean, which are helpful for the analysis of the performance of underwater acoustic communication systems. An underwater acoustic channel impulse response usually has a comb structure due to the multipath effect, suggesting a multipath channel model for acoustic communication simulations [16].

To analyze the performance of MCSK-FrFDH system in underwater acoustic channels, measured underwater acoustic channels can be used for modeling time-invariant multipath channels and time-variant multipath channels. Here we use the measured underwater acoustic channel from Wuyuan Bay, Xiamen in December, 2011. The average depth of Wuyuan Bay was about $6 \mathrm{~m}$, and the transmitter and receiver were placed at the depth of $3 \mathrm{~m}$ with a distance of about $700 \mathrm{~m}$. The source-receiver arrangement with the bay configuration shown in Fig. 5.

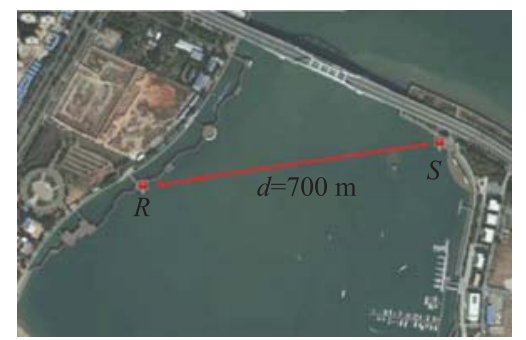

Fig. 5 Experimental arrangement of Wuyuan Bay, Xiamen

Fig. 6 illustrates the $250 \mathrm{~s}$ acoustic channel impulse response from Wuyuan Bay, and Fig. 7 shows the average of Fig. 6. One can see that the number of multipath is 5 and the maximum multipath delay is about $46 \mathrm{~ms}$. Table 1 summarizes the parameters of multipath, where data are normalized to the parameters of the first path. The statistical analysis indicates that the distribution of received signal conforms to Rayleigh distribution (see Fig. 8). 


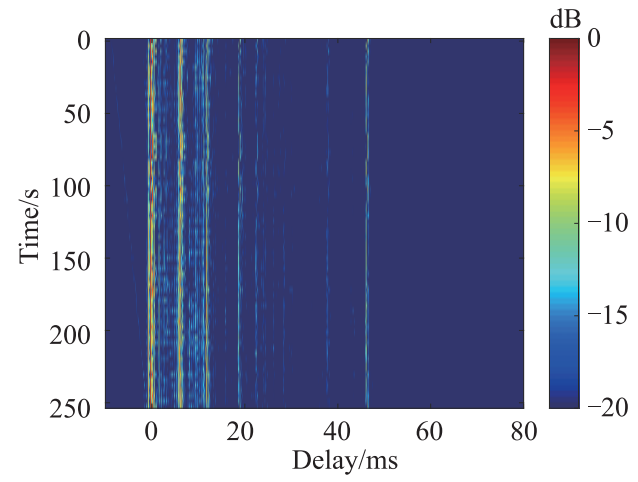

Fig. 6 Measured underwater acoustic channel impulse response from Wuyuan Bay, Xiamen

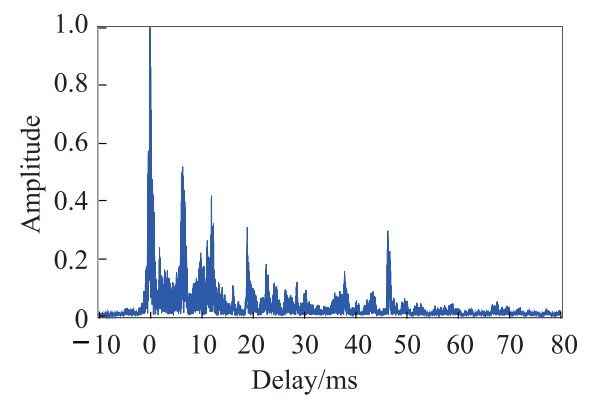

Fig. 7 Channel multipath structure of Wuyuan Bay, Xiamen

Table 1 Delay-amplitude parameters of Wuyuan Bay, Xiamen

\begin{tabular}{ccc}
\hline Path & Relative delay $/ \mathrm{ms}$ & Normalized amplitude \\
\hline 1 & 0 & 1.000 \\
2 & 6.4 & 0.524 \\
3 & 12.0 & 0.422 \\
4 & 18.9 & 0.312 \\
5 & 46.4 & 0.300 \\
\hline
\end{tabular}

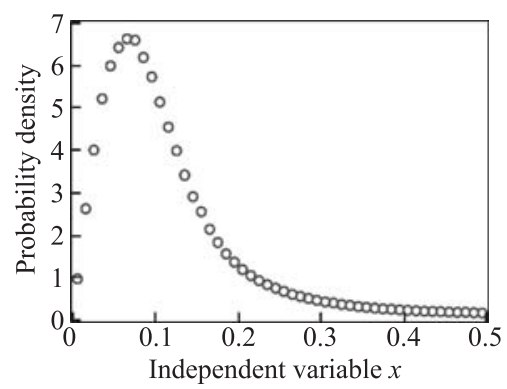

Fig. 8 Probability density of the envelope of received signal

\subsection{Parameter design}

To compare with the traditional MFSK frequency-hopped (MFSK-FH) system, the MCSK-FrFDH and MFSK-FH systems are designed to achieve the same anti-multipath ability $\tau_{m}$ and the data rate $R_{b}$. The sample rate $f_{s}$ is set to $48 \mathrm{kHz}$, and other parameters are listed in Table 2, including two settings for MCSK-FrFDH system. The first one has the advantage of long symbol length $T_{s}$ while the second one has the advantage of large chirp bandwidth $B_{c}$. The MFSK-FH system doubles the bandwidth $B$ to achieve the same data rate as the MCSK-FrFDH system. The selection of parameters should agree with the following principles:

(i) Hop count $H$, together with the product of $H$ and radix $M(M H)$ should be as small as possible in order to increase the FrFD/frequency-domain resolution and the initial frequency interval/frequency interval $\Delta f$.

(ii) The chirp bandwidth $B_{c}$ should be as large as possible for enlarging the difference between frequency modulation rates.

(iii) For security and anti-interference, hop count $H$ should be as large as possible.

Table 2 Parameters of MCSK-FrFDH system and MFSK-FH system

\begin{tabular}{cccc}
\hline Parameter & \multicolumn{2}{c}{ MCSK-FrFDH } & MFSK-FH \\
\hline$H$ & 3 & 4 & 8 \\
$M$ & 16 & 8 & 8 \\
$f_{s} / \mathrm{kHz}$ & 48 & 48 & 48 \\
$B / \mathrm{kHz}$ & $13-18$ & $13-18$ & $10-20$ \\
$\Delta f / \mathrm{Hz}$ & 100 & 133.33 & 156.25 \\
$B_{c} / \mathrm{Hz}$ & 200 & 733.33 & - \\
$T_{s} / \mathrm{ms}$ & 20 & 15 & 7.5 \\
$\tau_{m} / \mathrm{ms}$ & 60 & 60 & 60 \\
$R_{b} / \mathrm{bps}$ & 400 & 400 & 400 \\
\hline
\end{tabular}

It is difficult to satisfy all these principles in one MCSKFrFDH system. We should consider all the factors including the real acoustic channel in order to choose appropriate parameters when designing the system.

When hopped count $H=4$, the short time Fourier transform (STFT) and short time fractional Fourier transform (STFrFT) [17] of the transmitted signal of MCSKFrFDH system within the duration of $H T_{s}$ are shown in Fig. 9 and Fig. 10, respectively.

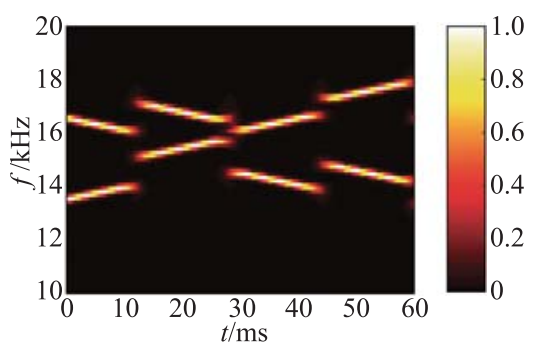

Fig. 9 STFT of the transmitted signal during one complete frequency-hopped pattern $(H=4)$

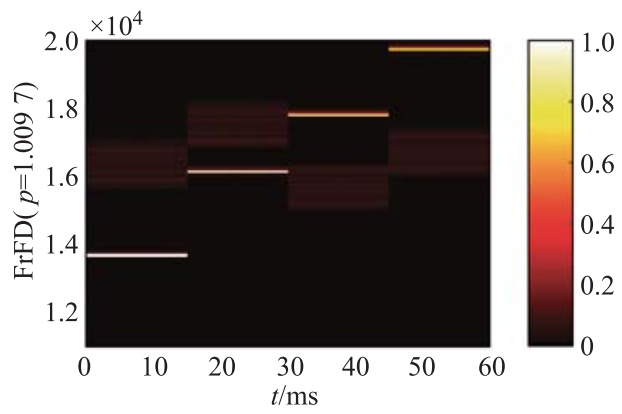

(a) STFrFT ( $p=1.0097$ ) of the subcarrier with positive frequency modulation rate 


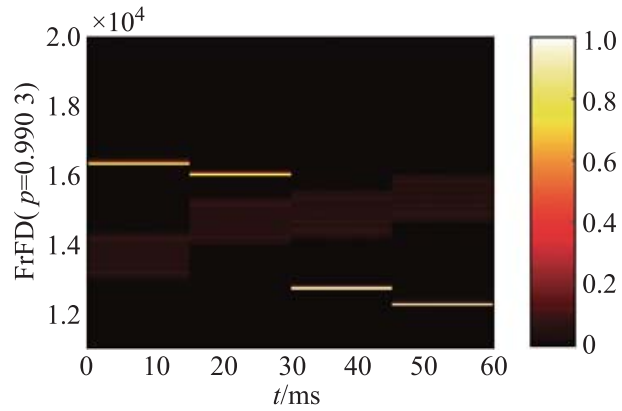

(b) STFrFT ( $p=0.9903$ ) of the subcarrier with negative frequency modulation rate

Fig. 10 STFrFT of the transmitted signal during one complete frequency-hopped pattern $(H=4)$

Fig. 9 and Fig. 10 indicate that signals overlap in frequency-domain can be separated easily in FrFD. One carrier component may be concentrated in FrFD, and the other may be expanded and attenuated, which does not affect the detection of the former one.

\subsection{Simulation results}

To assess the performance of MCSK-FrFDH system and MFSK-FH system, simulations are conducted in an additive white Gaussian noise (AWGN) channel, a timeinvariant multipath channel and a time-variant multipath channel. Two types of MCSK-FrFDH system proposed in Table 2 are denoted as 16CSK-3FrFDH and 8CSK4FrFDH respectively.

In the AWGN channel, the BER curves of the MCSKFrFDH system and the MFSK-FH system are shown in Fig. 11, indicating that the MCSK-FrFDH system has a better performance than the MFSK-FH system. Though the chirp bandwidth used in the 16CSK-3FrFDH system is smaller than that in the 8CSK-4FrFDH system, the long symbol length makes up the disadvantage of small difference between frequency modulation rates, and makes the 16CSK-3FrFDH system perform better.

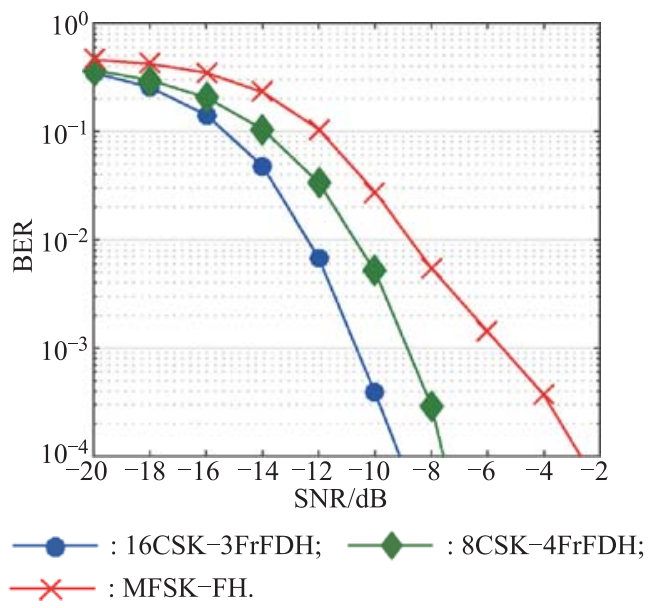

Fig. 11 BER curves of different systems in AWGN channel
Fig. 12 shows the BER curves for the 16CSK-3FrFDH and $8 \mathrm{CSK}-4 \mathrm{FrFDH}$ systems when the Doppler shift is present. Comparing it to Fig. 11, the BER curve of 16CSK3FrFDH system with the frequency shift $\left|\Delta f_{D o p}\right|=10 \mathrm{~Hz}$ is almost as same as the case without Doppler shifts, so is the BER curve of 8CSK-4FrFDH system with $\left|\Delta f_{D o p}\right|$ being $16 \mathrm{~Hz}$. When $\left|\Delta f_{D o p}\right|$ increases, the performance decreases with it. In other words, the maximum antiDoppler shifts of 16CSK-3FrFDH system and 8CSK4FrFDH system are respectively $10 \mathrm{~Hz}$ and $16 \mathrm{~Hz}$, both of which are much higher than the possible Doppler shifts in Wuyuan Bay, Xiamen [18].

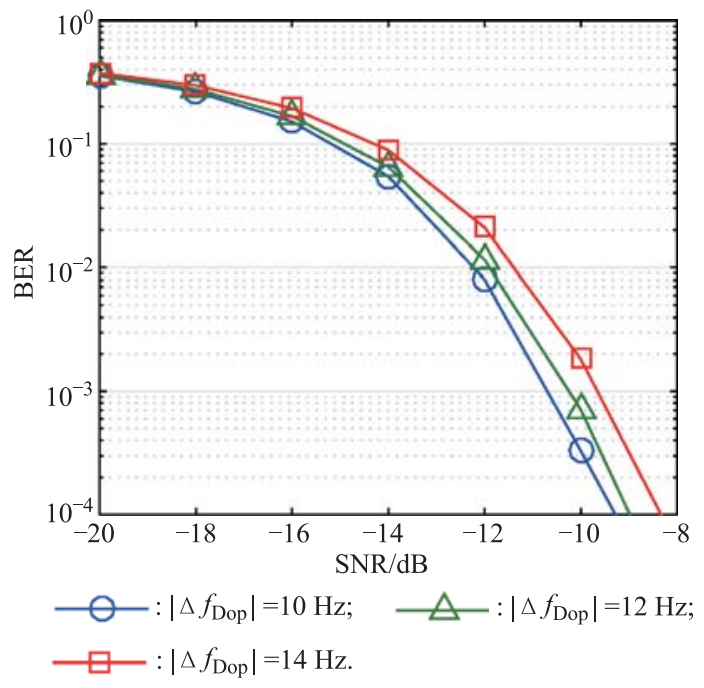

(a) BER curves of $16 \mathrm{CSK}-3 \mathrm{FrFDH}$ system

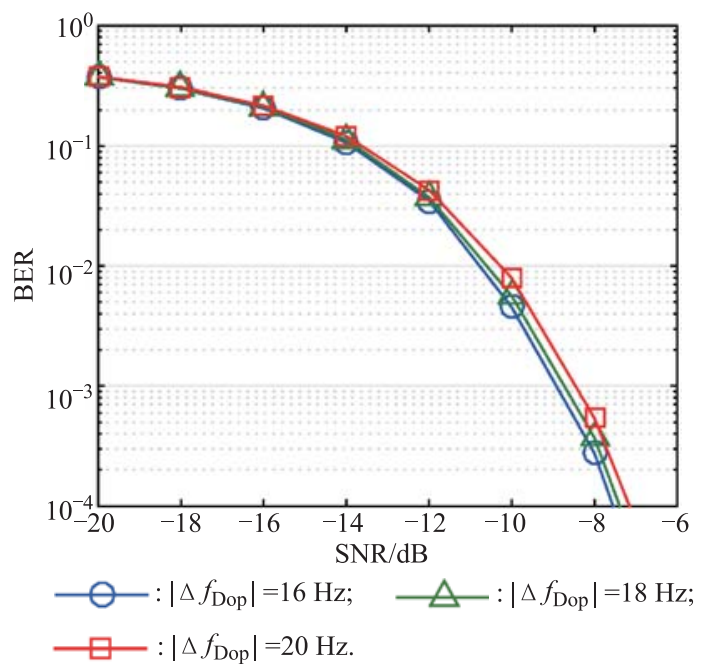

(b) BER curves of 8CSK-4FrFDH system

Fig. 12 BER curves of different systems with Doppler shifts

Fig. 13 exhibits the BER curves in a time-invariant multipath channel (the parameters of multipath are shown in Table 1), where the MCSK-FrFDH system performs better than the MFSK-FH system. When SNR $=-2 \mathrm{~dB}$, the 
BERs of 16CSK-3FrFDH system and 8CSK-4FrFDH system are $10^{-4}$ and $4 \times 10^{-4}$ respectively, while the BER of MFSK-FH system is $7 \times 10^{-3}$.

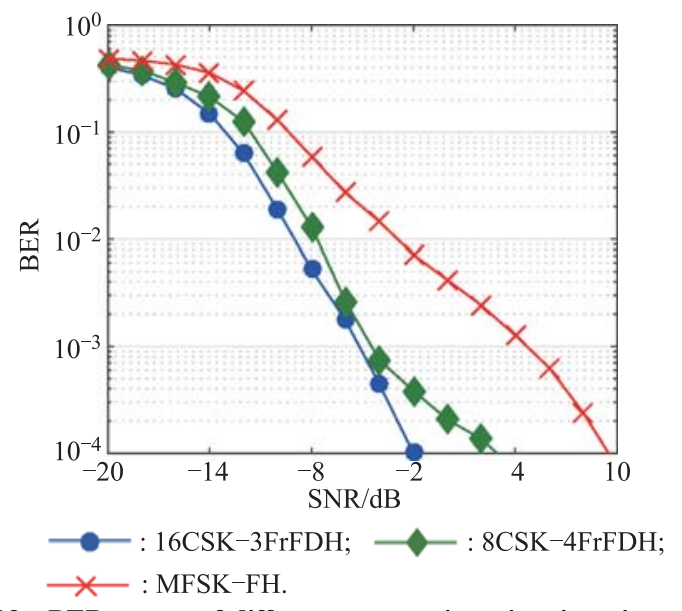

Fig. 13 BER curves of different systems in a time-invariant multipath channel

In a time-variant multipath channel (Fig. 6), those three systems all have error floors when SNR is above $8 \mathrm{~dB}$, and MCSK-FrFDH system is still better than MFSK-FH system as Fig. 14 shows. At low SNR, 16CSK-3FrFDH system performs better than 8CSK-4FrFDH system because of its higher FrFD resolution. At high SNR, the disadvantage of 16CSK-3FrFDH system, i.e., small difference between frequency modulation rates, emerges gradually while $8 \mathrm{CSK}-4 \mathrm{FrFDH}$ system is still able to resist the fading of amplitude and frequency caused by timevariant channel for its larger chirp bandwidth, making the BER of 8CSK-4FrFDH system ten times lower than that of 16CSK-3FrFDH system. Therefore, in practical scenarios, we can choose a suitable system according to the effect of real channel. For instance, if SNR is small, we can choose the 16CSK-3FrFDH system; otherwise, choose the 8CSK4FrFDH system.

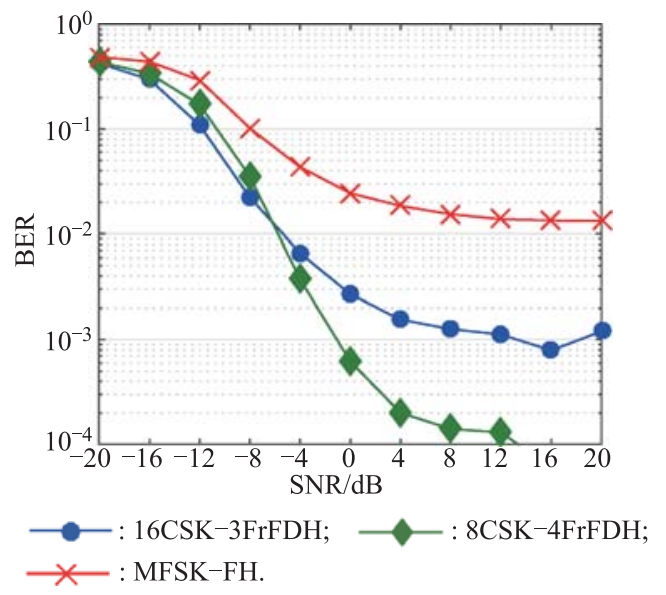

Fig. 14 BER curves of different systems in the time-variant multipath channel of Wuyuan Bay, Xiamen

\subsection{Ocean experiment}

In May 2016, MCSK-FrFDH and MFSK-FH were tested in an underwater acoustic communication experiment at Wuyuan Bay, Xiamen. The arrangement of transmitter and receiver (whose frequency band is $13-18 \mathrm{kHz}$ ) is the same as that in Fig. 5. The parameters of MCSK-FrFDH system are identical to those in Table 2, and the data rates of MFSK-FH system vary from $100 \mathrm{bps}$ to $400 \mathrm{bps}$. In the experiment, $10^{5}$-bit data are transmitted, and BERs at the receiver side are listed in Table 3.

Table 3 BERs in the experiment of Wuyuan Bay, Xiamen

\begin{tabular}{ccc}
\hline MFSK-FH & $\begin{array}{c}\text { 16CSK-3FrFDH } \\
(400 \mathrm{bps})\end{array}$ & $\begin{array}{c}\text { 8CSK-4FrFDH } \\
(400 \mathrm{bps})\end{array}$ \\
\hline $0.0023 @ 100 \mathrm{bps}$ & 0.00085 & 0.00056 \\
$0.007119200 \mathrm{bps}$ & 0.00136 & 0.00495 \\
$0.0332 @ 400 \mathrm{bps}$ & 0.00027 & 0.00052 \\
\hline
\end{tabular}

Two conclusions can be drawn from Table 3. First, the BER of MCSK-FrFDH system is about the order of $10^{-3}$ at 400-bps data rate, which is ten times lower than that of MFSK-FH system. Second, the data rate of MFSK-FH system has to reduce to below $100 \mathrm{bps}$ in order to achieve the BER $10^{-3}$. In other words, MCSK-FrFDH system quadruples the data rate of MFSK-FH system with the same bandwidth. In general, MCSK-FrFDH system improves the bandwidth efficiency of MFSK-FH system, revealing its great development potential in underwater acoustic communication.

\section{Conclusions}

In this paper, an FrFD frequency-hopped communication method is proposed and the underwater acoustic MCSKFrFDH system is designed based on this method. We derive the parameters of MCSK-FrFDH system including FrFD hopped pattern, minimum FrFD hopped interval and anti-Doppler shifts ability. The simulation results indicate that MCSK-FrFDH system uses only half bandwidth of MFSK-FH system but performs better at the same data rate and anti-multipath capability. While the experiment results reveal that MCSK-FrFDH system quadruples the data rate of MFSK-FH system with the same bandwidth, and decreases the BER ten times lower than that of MFSK-FH system at the same data rate. This study offers an alternative method to improve bandwidth efficiency for underwater acoustic frequency-hopped communications, i.e., FrFD hopping in two FrFDs without interference. Future researches for underwater acoustic frequency-hopped communications may include FrFD hopping in more than two FrFDs for higher band efficiency.

\section{References}

[1] J. G. Proakis, M. Salehi. Fundamentals of communication systems. New Jersey: Prentice Hall PTR, 2007. 
[2] L. Freitag, M. Grund, C. V. Alt, et al. A shallow water acoustic network for mine countermeasures operations with autonomous underwater vehicles. Underwater Defense Technology, 2005: 1-6.

[3] A. Baggeroer. An overview of acoustic communications from 2000-2012. Underwater Communications: Channel Modelling \& Validation, 2012, 5: 201-207.

[4] P. P. Beaujean, M. Pajovic, E. Carlson, et al. Frequencyhopped frequency division multiplexed signaling for underwater acoustic communications between 60 and $90 \mathrm{kHz}$ in ports and very shallow waters. Proc. of the OCEANS MTS/IEEE Biloxi-Marine Technology for Our Future: Global and Local Challenges, 2009: 1-7.

[5] F. Hou. Research on underwater acoustic FH communication with independent multi-source parallel transmission. Xiamen: Xiamen University, 2013. (in Chinese)

[6] H. M. Ozaktas, O. Arikan, M. A. Kutay, et al. Digital computation of the fractional Fourier transform. IEEE Trans. on Signal Processing, 1996, 44(9): 2141-2150.

[7] S. C. Pei, J. J. Ding. Closed-form discrete fractional and affine Fourier transforms. IEEE Trans. on Signal Processing, 2000, 48(5): $1338-1353$.

[8] Y. Chen. Research on application of fracional Fourier transform in underwater acoustic communication. Harbin: Harbin Engineering University, 2012. (in Chinese)

[9] X. J. Sha, X. Tang, X. Y. Ning, et al. Method and device for transmitting and receiving fractional Fourier domain - frequency domain-frequency-hopped signals. CN 200810064381, 2012. (in Chinese)

[10] X. J. Sha, X. Tang, R. H. Wen, et al. A method for generating and receiving fractional Fourier domain-frequency-hopped signals. CN 200810064162, 2012. (in Chinese)

[11] X. J. Sha, J. Shi, Q. Y. Zhan. Fractional Fourier transform theory and its applications in communication systems. Beijing: Posts and Telecommunications Press, 2013. (in Chinese)

[12] W. J. Wang. Research of chirp underwater spread spectrum communication technology based on FRFT. Xiamen: Xiamen University, 2014. (in Chinese)

[13] R. Tao, B. Deng, Y. Wang. Fractional Fourier transform and its applications. Beijing: Tsinghua University Press, 2009.

[14] X. B. Tu, X. M. Xu, L. L. Yang, et al. Application of FRFT in time-delay and frequency-shift joint estimation for underwater acoustic channels. Systems Engineering and Electronics, 38(7): 1666-1672. (in Chinese)

[15] X. H. Zhao, B. Deng, R. Tao. Dimensional normalization in the digital computation of the fractional fourier transform. Journal of Beijing Institute of Technology, 2005, 25(4): 360-364. (in Chinese)

[16] W. H. Tranter. Principles of communication systems simulation with wireless applications. New Jersey: Prentice Hall PTR, 2004.

[17] R. Tao, Y. L. Li, Y. Wang. Short-time fractional Fourier transform and its applications. IEEE Trans. on Signal Processing, 2010, 58(5): $2568-2580$.

[18] X. M. Xu. Studies on data transmission techniques in shallow water acoustic channels. Xiamen: Xiamen University, 2002. (in Chinese)

\section{Biographies}

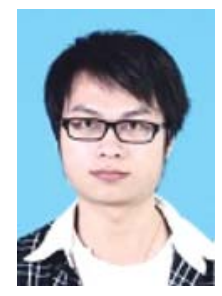

Xingbin Tu was born in 1989. He graduated in marine sciences from College of Ocean and Earth Science, Xiamen University, in 2012. He is currently a Ph.D. candidate in Xiamen University. His research interests include underwater acoustic communication and signal processing.

E-mail: xbtu@stu.xmu.edu.cn

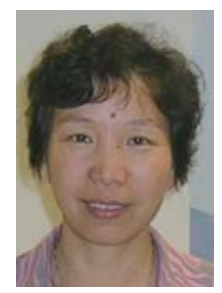

Xiaomei Xu was born in 1960. She graduated from Department of Oceanography, Xiamen University, in 1982. She received her M.S. degree in underwater acoustic telemetry in 1988 and Ph.D. degree in underwater communication from Xiamen University in 2002. During 1994 and 1995, she studied in Oregon State University (USA). She is now a professor in College of Ocean and Earth Science, Xiamen University. Her research interests include underwater communication, underwater acoustic telemetry and underwater acoustic network. E-mail: xmxu@xmu.edu.cn

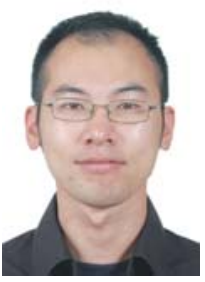

Zheguang Zou was born in 1985 . He received his B.S. and M.S. degrees in marine sciences from College of Ocean and Earth Sciences, Xiamen University, in 2008 and 2011, respectively. Currently, he is a $\mathrm{Ph} . \mathrm{D}$. candidate in oceanography through the dual-degree program established by the Joint Institute for Coastal Research and Management (JointCRM), between the University of Delaware (USA) and Xiamen University (China). His research interests are acoustical oceanography, shallow-water acoustic wave propagation, and underwater acoustic communication.

E-mail: zzg@udel.edu

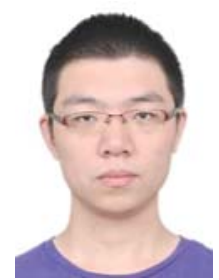

Liangliang Yang was born in 1985. He received his B.S. and M.S. degrees in electronic and information engineering in 2009 and 2012. Then he worked one year in Shanxi Meteorological Bureau before enrolling in the doctoral program at Xiamen University, where he majored in underwater acoustics. $\mathrm{He}$ is currently studying as a visiting Ph.D. student in Dove Marine Laboratory at Newcastle University (UK). His research focuses mainly on anthropogenic \& biological sound, sound propagation, and signal processing.

E-mail: yll@stu.xmu.edu.cn

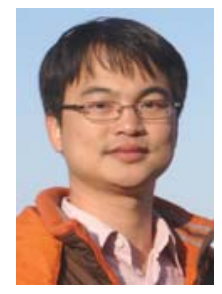

Jianming Wu was born in 1978. He graduated in marine sciences from College of Oceanography and Environmental Science, Xiamen University, in 2001. He is currently an engineer in College of Ocean and Earth Science, Xiamen University. His research interests include underwater acoustic communication and signal processing.

E-mail:wjm@xmu.edu.cn 